16 | 2020

Les marges créatrices : intellectuel.le.s afrodescendant.e.s et indigènes auX Amériques, XIX-XXe siècle

\title{
Du Cricket, de la Révolution mondiale et de Trinidad : jeunesse caribéenne de C. L. R. James
}

About Cricket, World Revolution and Trinidad: C. L. R. James's Caribbean Youth by

Acerca del Cricket, de la Revolución Mundial y de Trinidad : la juventud caribeña de C. L. R. James

\section{Olivier Maheo}

\section{OpenEdition}

\section{Journals}

Édition électronique

URL : http://journals.openedition.org/ideas/860

DOI : 10.4000/ideas.8601

ISSN : 1950-5701

Éditeur

Institut des Amériques

Référence électronique

Olivier Maheo, «Du Cricket, de la Révolution mondiale et de Trinidad : jeunesse caribéenne de C. L. R. James », IdeAs [En ligne], 16 | 2020, mis en ligne le 01 octobre 2020, consulté le 18 octobre 2020. URL : http://journals.openedition.org/ideas/8601 ; DOI : https://doi.org/10.4000/ideas.8601

Ce document a été généré automatiquement le 18 octobre 2020

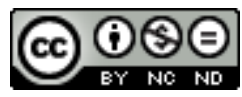

IdeAs - Idées d'Amériques est mis à disposition selon les termes de la licence Creative Commons Attribution - Pas d'Utilisation Commerciale - Pas de Modification 4.0 International. 


\section{Du Cricket, de la Révolution mondiale et de Trinidad : jeunesse caribéenne de C. L. R. James}

About Cricket, World Revolution and Trinidad: C. L. R. James's Caribbean Youth by

Acerca del Cricket, de la Revolución Mundial y de Trinidad : la juventud

caribeña de C. L. R. James

Olivier Maheo

« Le cricket c'est le cricket dans vos ricochets, mais vu de loin cela ressemble à de la politique».

"Is cricket is cricket in yuh ricketics but from far it

look like politics"

(Agard, 1994 : 546-548).

Ce poème est dédié à C. L. R. James.

\section{Introduction}

1 Ce numéro de la revue Ideas propose d'interroger les marges créatrices, en montrant comment une situation marginale, qu'elle soit géographique, sociale, ethnoraciale, politique, ou tout à la fois, peut s'avérer féconde d'un point de vue intellectuel. Lorsque l'on élargit la focale à l'échelle de l'Amérique, y compris la Caraïbe, les rapports entre marges et centres se trouvent bouleversés - en ce qui concerne les Antilles britanniques, le centre, excentré, est situé à Londres. Il nous a semblé que la personne de Cyril Lionel Robert James personnifiait ces traversées de frontières de la marge au centre et inversement, tout d'abord physiquement, de Trinidad où il est né, à l'Angleterre où il émigra, aux Etats-Unis où il vécut, et à Trinidad où il revint ${ }^{1}$. Il nous paraît aussi que les interrogations qu'il soulève dans ses premiers écrits remettent en question les notions mêmes de marges et de centre, dans la perspective d'une analyse 
qui, à la suite d'Homi Bhabha, renonce au paradigme colonial pour penser, à l'encontre d'identités fixes, l'hybridation et le «tiers-espace [qui] quoi qu'irreprésentable en soi, constitue les conditions discursives d'énonciation qui attestent que le sens et les symboles culturels n'ont pas d'unité ou de fixité primordiales, et que les mêmes signes peuvent être appropriés, traduits, réhistoricisés et réinterprétés " (Bhabha H., 2007 : 30).

2 James est né à Tunapuna, sur l'île de Trinidad en 1901. Il a vécu essentiellement aux Etats-Unis, puis en Grande-Bretagne. Ses articles et ses ouvrages abordent de très nombreux sujets : depuis l'histoire des Caraïbes en passant par l'art, la littérature, la lutte de classe, la musique et le cricket. Il fut avec passion historien, auteur dramatique et journaliste sportif. Son ouvrage sans doute le plus connu demeure Les Jacobins Noirs (1949), qui pourrait être considéré comme un premier pas précoce vers l'écriture d'une histoire mondiale, bien avant la World History anglo-saxonne des années 1980, ou l'histoire globale des années 1990. Il ne s'agit pas en effet simplement d'une histoire de Saint-Domingue, mais d'un récit des interconnexions entre la révolution française et les événements caribéens. Cette histoire entrelacée, caribéenne, représente une forme d'écriture diasporique qui annonce le concept d'Atlantique noir et représente les premiers pas en direction d'une écriture non-européenne du monde atlantique. Les Jacobins Noirs ont donné lieu à de nombreux articles et travaux, et ce succès est peutêtre en partie responsable du relatif effacement d'autres ouvrages de James, pièces de théâtre, fictions diverses, et essais politiques. A ses déplacements physiques fréquents et parfois contraints comme lorsqu'il est expulsé des Etats-Unis en 1952, on pourrait ajouter les traversées de frontières disciplinaires, de la littérature à l'histoire et réciproquement, et son cheminement intellectuel, d'une éducation britannique à une pensée marxiste et anticolonialiste originale. Ses écrits sont innombrables, tout comme les travaux à son propos. Il a été le sujet d'au moins cinq biographies et il est l'objet d'une véritable redécouverte depuis une dizaine d'années (Rosengarten F., 2007; Buhle P., 1988; Henry P. et al., 1992; Renault M., 2016; Worcester K., 1996; Høgsbjerg C., 2014). Cela pourrait sembler une gageure d'ajouter quelques lignes mais nous voulons nous pencher sur la période de sa vie peut-être la moins éclairée, celle d'un jeune intellectuel noir à Trinidad dans les années 1920, qui met précisément en lumière les relations complexes entre marges et centres. Ces années ont sans doute été un peu oubliées du fait de l'écho important que ses écrits ultérieurs rencontrèrent, à commencer par les Jacobins Noirs ${ }^{2}$. James, qui a été formé selon les principes d'une éducation victorienne examine dans ses premiers écrits, y compris des fictions, les conflits de statut, d'identités culturelles figées. A l'inverse il met en avant les thèmes du passage, de la transition, de la traduction, qui permettent, ainsi que l'historien Homi Bhabha l'a proposé dans les années 1980 , de « déplacer les conceptions traditionnelles d'analyse des situations coloniales en termes d'exploitation et de domination et déployer une analyse critique des oppositions réifiées entre centre et périphérie, identité et altérité qui les rigidifient dans des concepts stériles » (Cuillerai, M., 2010).

3 Avant qu'il ne découvre le marxisme en Grande-Bretagne en 1932 et qu'il ne l'étudie et n'y adhère aux côtés du mouvement trotskyste dans un premier temps, ce que nous n'aborderons que marginalement ici, son parcours intellectuel le conduit d'abord vers l'anticolonialisme. Certains ont vu un paradoxe dans la façon dont James, partisan du self-government, se définit tout d'abord en termes eurocentrés, affirme son amour du 
cricket, de Shakespeare, de Melville et de la philosophie classique. Sylvia Wynter, romancière, dramaturge et essayiste jamaïcaine résume ainsi cette identité plurielle :

Noir et pourtant britannique, sujet colonial à la naissance mais cependant formé dans les codes des Public Schools anglaises, colonial natif mais culturellement intégré dans le code de l'école publique, solidaire de la cause du prolétariat mais membre de la classe moyenne, marxiste et puritain, un intellectuel grand joueur de cricket, Africain par ses origines mais occidental, trotskyste et panafricaniste, marxiste mais défenseur des Black Studies, Noir comme la majorité des Antillais mais aussi Noir comme la minorité noire américaine.

(Wynter S. $1992: 69)^{3}$

4 Tout cela a été déjà débattu, et nous suivrons Paul Buhle, selon qui ces contradictions ne sont que superficielles (Buhle P., 2003). Après tout le cricket, sport impérial, est luimême devenu un vecteur d'affirmation nationale pour les jeunes nations décolonisées. Cette analogie permet d'aborder la complexité de la relation coloniale. Nous nous appuierons pour notre part sur deux ouvrages de jeunesse qu'il publia après son arrivée à Londres : le court roman Minty Alley, écrit à la fin des années 1920, et sa biographie d'un leader politique trinidadien, The Life of Captain Cipriani (James C. L. R., 1936 ; 1932).

\section{Classiques et cricket : une éducation britannique}

\section{De la fenêtre de Tunapuna}

5 James n'a pas écrit d'autobiographie au sens strict, mais Beyond a Boundary (1963) contient de nombreux éléments biographiques ${ }^{4}$. Dans le premier chapitre, James se place sous un triple héritage : celui du puritanisme, hérité de grands-parents venus d'autres îles anglophones, de son amour des livres, et du cricket. Le thème de l'héritage familial est répété à l'envi et les descriptions des grands-pères, de la mère et du père visent à expliquer la fermeté de caractère et l'indépendance d'esprit dont lui-même fait preuve. Les parents de James appartenaient à la première génération de Noirs nés après l'émancipation. Son père était enseignant (James C.L. R., et al., 1992: 506). James propose une analyse du succès du cricket dans le contexte colonial, un sport qui " était arrivé à maturité du fait de deux facteurs : l'ascension d'une classe moyenne noire, et les primes élevées versées aux joueurs des équipes anglaises » (Ibid. : 10). La fenêtre de sa chambre d'enfant s'ouvre sur le terrain de cricket, et il passe des heures à observer les matchs. James explique la popularité d'un sport britannique dans les colonies par le fait que le cricket « est un jeu de haute et difficile technique. S'il ne l'était pas, il ne pourrait pas porter la charge sociale et les implications qu'elle comporte » (Ibid. : 34$)^{5}$. Il s'agit sur le terrain de mettre en scène les « rivalités de classe et de race [qui] étaient trop intenses. [...] Ainsi, le terrain de cricket était une scène sur laquelle des individus sélectionnés jouaient des rôles représentatifs chargés d'une signification sociale" (Ibid.: 88)6.

Excellent élève, il obtient en 1911 une place de boursier dans le prestigieux Queen's Royal College, un établissement secondaire qui imite dans son fonctionnement les Grammar Schools britanniques, ces écoles dans lesquelles les élèves n'étaient admis qu'après une sélection très stricte, et qui était réservé, de facto et non de jure, aux enfants de l'élite. Il y étudie le grec ancien, le latin, la littérature anglaise et française. Il y reçoit, dit-il, « une éducation excellente pour me préparer à devenir un intellectuel britannique » (Ibid. : 65) . 
7 Avec ce même ton ironique, il décrit la voie toute tracée par ses parents, vers les plus hautes ambitions qu'un Noir de la classe moyenne pouvait alors nourrir à Trinidad, où les postes les plus qualifiés et les plus prestigieux étaient réservés à la minorité blanche, qui possédait alors l'essentiel des capitaux et des plantations. Seules les études de médecine ou de droit pouvaient permettre à un Noir de s'élever socialement, le succès ultime étant d'être choisi par le gouverneur de cette colonie de la Couronne comme l'un des rares représentants noirs au sein du conseil législatif, dont les membres n'étaient pas élus jusqu'en $1925^{\circ}$. Cependant, en ce début des années 1920 , James ne remet en question ni la situation coloniale, ni la stratification raciale qui recouvre pour l'essentiel les divisions sociales. Il s'intéresse avant tout à la littérature, et au cricket. Il se signale comme un élève excellent et turbulent, menacé à plusieurs reprises de renvoi de son école.

Ce n'est pas toutefois la discipline scolaire mais le cricket qui lui fait découvrir l'injustice : son père, un excellent joueur, a dû renoncer à ses ambitions sportives pour subvenir aux besoins de sa famille9. Par ailleurs un ami d'enfance, Learie Constantine (1901-1971), entame à partir de 1920 une brillante carrière de joueur professionnel. Lorsqu'il est en tournée en Grande-Bretagne, Constantine se voit refuser l'accès à certains hôtels, parce qu'il est noir. James écrit à son propos qu'il :

S'était rebellé contre le fossé révoltant entre sa place de joueur de cricket de premier rang, et son statut de troisième catégorie en tant qu'humain. Les limites qui lui étaient imposées du fait des conditions sociales dans les Antilles lui devinrent intolérables et il décida de ne plus les supporter.

(Ibid., p. 106) ${ }^{10}$

De son côté James est devenu enseignant en 1918, d'abord dans une école privée, puis dans le même Queen's Royal College où il avait étudié.

\section{Platon sous les tropiques ${ }^{11}$}

Son éducation classique, grec, latin, humanités, n'a rien à envier à celle reçue dans les meilleures écoles anglaises ${ }^{12}$. Il a le sentiment de participer autant à la civilisation occidentale, voire bien mieux, que la plupart des Blancs de Trinidad. Il définit les Trinidadiens comme des Britanniques avant tout et affirme sa valeur littéraire du fait de sa culture classique. Il n'utilise jamais le terme de créolisation, mais décrit plutôt une culture britannique transplantée sous les tropiques. A partir de 1925, il commence à développer une conscience politique.

11 Sa première passion littéraire est pour l'auteur victorien Thackeray et la Foire aux vanités dont l'attention prêtée aux ridicules des puissants est pour lui une source inépuisable au service de la critique sociale et un modèle de style (Thackeray W. M., 1847-1848). Il écrit ainsi: "Je riais sans cesse des blagues de Thackeray, de ses ricanements et de ses railleries sur l'aristocratie et les gens haut placés. C'est Thackeray, et non Marx, qui m'a le plus influencé » (Ibid. : 39) ${ }^{13}$.

12 Cette formation classique participe d'un effort pour se préparer méthodiquement à devenir un auteur, tout d'abord de fiction. Il publie dans la presse des nouvelles, en même temps qu'il rédige en 1928 le manuscrit de son unique roman, Minty Alley (1936). 


\section{La littérature de l'arrière-cour (barrack-yard literature) : le Beacon Group}

\section{Mauvais genre} ou créoles, échangent autour de leurs expériences d'écriture. Ils forment un cercle littéraire d'abord informel, qui prend le nom de Beacon (le phare) en $1930^{14}$. James publie cinq nouvelles qui participent à définir ce genre littéraire local ${ }^{15}$. Il a l'idée d'un magazine qui publierait leurs textes et il y associe Ralph de Boissière (1907-2008) et Alfred Mendes (1897-1991) (de Boissière, 2001, 12). Un créole d'origine portugaise, Albert Gomes (1911-1978) finance la publication d'une première revue littéraire nommée Trinidad dont seuls deux numéros paraissent, avant qu'elle se transforme en un mensuel à partir de 1931, The Beacon, qui parait vingt-huit fois entre 1931 et $1933^{16}$.

Leurs premières fictions dénoncent l'hypocrisie de l'élite blanche, qui prétend s'élever au-dessus de la supposée vie immorale des classes populaires. Dans Boodhoo, Mendes s'en prend à l'immoralité des plus riches, qui reprochent à leurs salariés de ne pas être mariés, mais les paient trop peu pour qu'ils puissent l'envisager (Mendes A. H., 1932). Albert Gomes ne cache pas sa sympathie pour l'URSS, et décrit ce groupe de jeunes hommes en colère comme le foyer d'un mouvement d'éveil intellectuel ${ }^{17}$. Tous défendent une forme de littérature populaire, plus tard surnommée Barrack-yard Literature, dont l'action se déroule dans les quartiers pauvres de Port-of-Spain; ce genre tire son nom des cours collectives des bidonvilles de Port-of-Spain. Ces espaces partagés regroupent des rangées de chambres alignées dont souvent les cloisons n'allaient pas jusqu'au plafond. Ces quartiers étaient associés à la population noire la plus pauvre, dont culture dite jamette avait donné naissance au XIX siècle au calypso et au carnaval. Nous retrouvons ici le thème des marges, puisqu'il faut remarquer que le terme jamette vient de diamètre, et indiquait la position périphérique de ceux qui participent de cette culture. Ces quartiers avaient été au cœur des émeutes des années 1919-1920. Nous proposons ci-dessous une photographie de ces logements exigus qui donnaient sur l'espace clos d'une cour intérieure, lieu d'une vie collective intense. James était fasciné par ces quartiers, et il y passait des heures. 


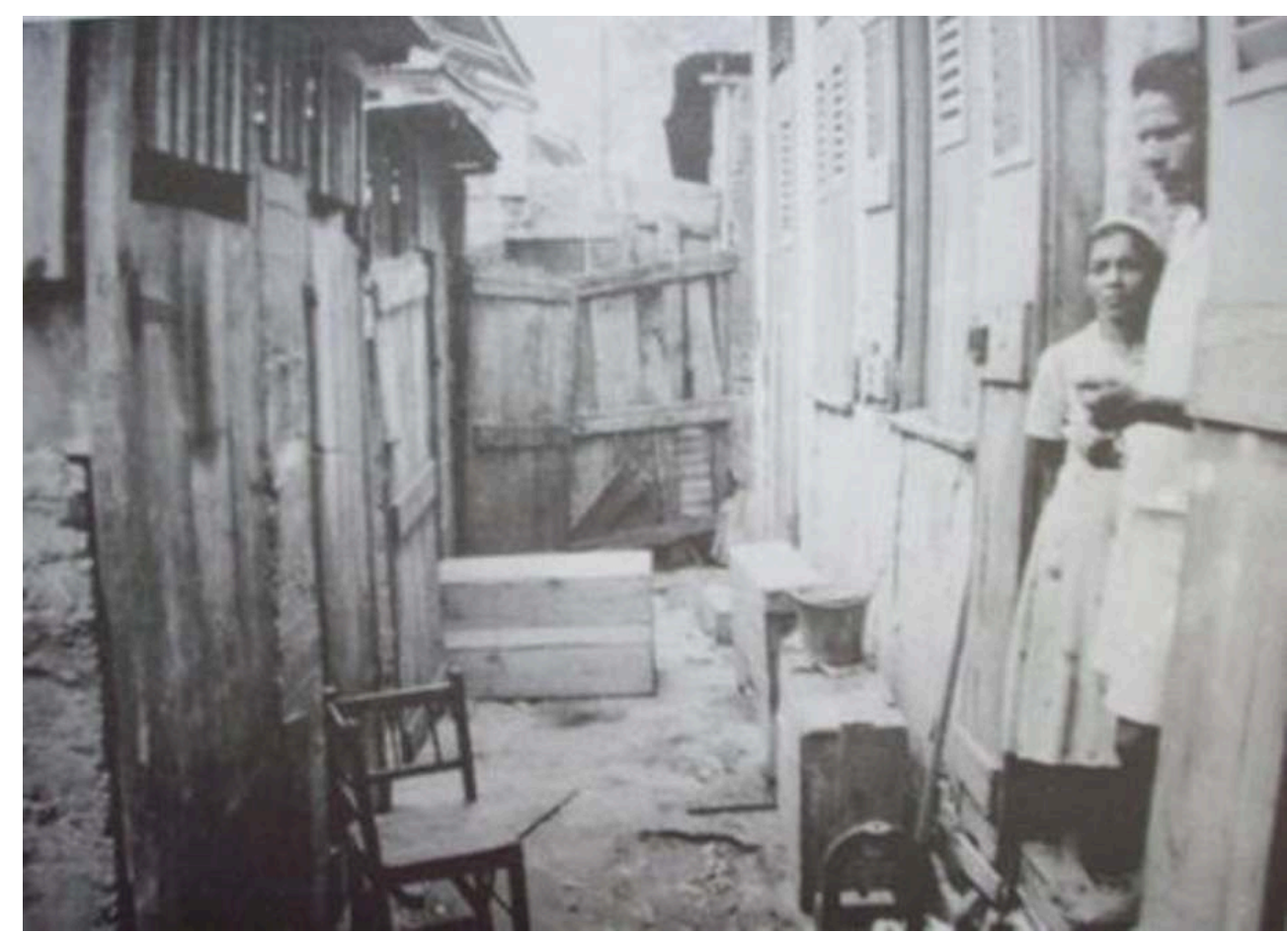

Figure : Barrack-yard, Port-Of-Spain en 1924.

Source: James Cummings, Barrack Yard Dwellers, St. Augustine Trinidad: School of Continuing Studies, The University of the West Indies, St. Augustine, Trinidad, 2004, 29.

Deux des nouvelles écrites par James, La Diablesse et The Star That Wouldn't Shine, prennent la forme de récits qu'il prétend extraire directement de ce qui lui a été raconté dans ses escapades dans les quartiers populaires ${ }^{18}$. On trouve dans ces écrits un intérêt quasi anthropologique pour la culture jamette, la vie des classes populaires et l'utilisation du langage populaire. Son unique roman, Minty Alley, rédigé en 1928, sera publié en en 1936 "par hasard " grâce à Frederic Warburg, de la maison Secker and Warburg, qui travaillait alors à l'édition de ce qui va devenir World Revolution (James C. L. R., 1937). Warburg, qui l'avait entendu parler de son unique roman, lui demanda son manuscrit et le publia (James C. L. R., $2000: 124$; Hall S. et al., $1995: 18)^{19}$. Minty Alley met en scène la difficulté d'un créole éduqué à s'adapter à la vie populaire.

\section{Le panoptique du ${ }^{\circ} 2$ de Minty Alley}

Le personnage principal de Minty Alley, Haynes, est un jeune Créole de bonne éducation dont la mère vient de mourir et qui, du fait de ses difficultés financières, est contraint de louer une chambre au $\mathrm{n}^{\circ} 2$ de Minty Alley. Haynes ne désirait qu'une chose, quitter l'île, mais le décès prématuré de sa mère l'en empêche. Sans argent et sans profession, il doit s'installer dans le quartier des barrack-yards mais il ne fait pas partie de ce milieu populaire. Cette cour est la scène ouverte dans laquelle les confidences et les conflits s'entremêlent. Les descriptions sont rares et l'essentiel du texte est construit autour de dialogues qui révèlent petit à petit les personnages et la complexité de leurs relations du fait des différences de classe sociale, de couleur et de culture.

17 James ne s'attarde pas sur la description psychologique du petit-bourgeois éduqué Haynes, dont le lecteur n'apprendra que peu de choses, hormis qu'il est le seul à avoir 
reçu une éducation dans le but de devenir employé de bureau. A son arrivée au ${ }^{\circ} 2$ il est incommodé par le vacarme et les odeurs qui viennent de la boulangerie que tient Mrs Rouse. Il ferme le plus souvent sa porte, mais il assiste aux drames quotidiens du $\mathrm{n}^{\circ} 2$ en regardant au travers d'une fissure dans son mur. Rapidement il s'emploie à élargir cette fissure avant que le voyeur ne soit finalement naturellement mêlé et absorbé par la vie de la cour. S'il peut observer ses voisins, c'est précisément parce qu'il n'appartient pas à cet univers, et la cour devient la métaphore d'un dispositif panoptique qui donnerait à voir toute la société trinidadienne. D'ailleurs c'est parce qu'il vient de s'y installer qu'il peut circuler librement entre les logements et les habitants. Cependant il ne peut lui-même échapper au dispositif d'observation, et il est aussi exposé aux regards. La propriétaire des lieux, Mrs Alice Rouse, se plaint auprès de lui du fait que son compagnon depuis 18 ans, Benoit, a une liaison avec une locataire, l'infirmière Mrs Jackson. On croise aussi une domestique indienne nommée Philomen, le fils de Mrs Jackson, Sonny, Ella, une autre domestique et toute une galerie de personnages plus ou moins pauvres, plus ou moins éduqués, qui tous se débattent pour survivre. Ce petit monde accorde rapidement à Haynes du respect et une place privilégiée du fait qu'il est un homme, créole, relativement aisé et éduqué. Mais il est bien mal préparé pour ces relations franches et pleines de vitalité, il manque de confiance en lui et juge les relations sociales à l'aune d'une morale conventionnelle qui n'a pas cours au n 2 (Parris E. D., 1981 : 98). Au bout du compte, son éducation le rend inapte à cette implication aussi bien émotionnelle que physique dans la vie de la classe ouvrière de Port-of-Spain. D'une certaine manière Haynes, déchiré entre son éducation et son lien avec son île, connait la même souffrance que l'intellectuel états-unien W.E.B. $\mathrm{Du}$ Bois a conceptualisé en 1903 sous l'expression de «double-conscience » (Chivallon C., 2008 ; Du Bois W. E. B., 1903).

18 Ainsi la position sociale intermédiaire de James, celle d'un intellectuel qui « va au peuple » en quête d'authenticité, de la réalité d'une possible identité trinidadienne, est mise en scène par le personnage de Haynes. James met ainsi en jeu son statut d'homme éduqué et sa position sociale, pour se placer sous le regard des classes populaires, arbitres en définitive de son devenir. Le dilemme de l'intellectuel, dont l'aliénation est d'abord liée à son éducation, peut être surmonté par cette interaction avec le peuple. Plus tard, James devenu marxiste en 1934 du fait de sa découverte des écrits historiques de Trotsky, oppose de la même façon la créativité de la classe ouvrière aux bavardages des intellectuels, y compris socialistes (James C. L. R., 1962 : 2). Ainsi en 1968, lors d'un congrès culturel à la Havane, il formule plus explicitement cette idée en proposant aux délégués de préparer la voie pour mettre fin aux prétentions des intellectuels à être les principaux vecteurs de la culture : «pour être véritablement conscient de leur rôle, ils devaient rejeter les pièges de la société occidentale, à commencer par la hiérarchie qui les place au-dessus des masses» (Hamilton C., 1992 : 439). En 1928, le héros Haynes abandonne sa chambre et se lance dans l'arène de la cour du $n^{\circ} 2$. Haynes n'est pas James, cependant ce dernier a raconté en 1972 comment il avait vécu volontairement quelques mois dans un barrack-yard avant d'écrire Minty Alley : il avoue avec candeur qu'il n'était à l'époque aucunement conscient des implications politiques de son récit (Sander L. S., et al., $1972: 33$ ).

19 L'essentiel de l'action se déroule dans la cour et ce qui se passe au dehors est le plus souvent rapporté par le récit d'un personnage. Le roman multiplie les péripéties mais toutes tournent pour l'essentiel autour du trio entre Mrs Rouse, son compagnon Benoit, et l'infirmière Jackson. Celle-ci est finalement arrêtée pour un vol de vêtements, et 
Benoit décède d'une attaque cardiaque. L'ensemble de la propriété de Mrs Rouse, le $\mathrm{n}^{\circ} 2$ de Minty Alley est hypothéqué car celle-ci ne parvient plus à payer son crédit. Ce roman apparait comme un hommage au petit peuple de Port-of-Spain, et particulièrement aux femmes qui sont la colonne vertébrale $d u n^{\circ} 2$, de son économie mais aussi de la grande famille que la cour réunit. Elles dominent les personnages masculins aussi bien par leur énergie, leur esprit de répartie, que par leur dignité et leur capacité à construire une vie supportable pour elles et tous les résidents de la cour (Kamugisha A., 2014 : 44). James cherche par son écriture à développer une voie originale, proprement trinidadienne mais aussi un style propre.

\section{La foire aux vanités antillaise}

Minty Alley est construit à la manière d'une pièce de théâtre autour de la petite scène représentée par la cour. Le roman rappelle par la vivacité des dialogues et la grande variété de personnages la Foire aux vanités, ce classique victorien que James connaissait quasiment par cœur (Thackeray W. M., 1847-48). Son sujet, essentiellement une description du petit peuple de la capitale, de ses conditions de vie et de logement, tout comme certains des procédés qu'il utilise, à commencer par la transcription du créole anglo-antillais, évoquent certains des codes de la littérature prolétarienne, cependant il ne s'agit pas d'un roman protestataire ou militant même si certains critiques l'ont analysé sous cet angle (Carby H. V., 1990). A ce sujet, un ami de James, Ralph de Boissière, a écrit au contraire que les canons littéraires qu'ils suivaient collectivement leur permettaient de représenter la souffrance des plus pauvres, mais qu'ils étaient collectivement incapables d'imaginer que ces gens puissent devenir les héros de leurs propres combats (de Boissière R., 2001: 16) ${ }^{20}$. Si James s'intéresse ainsi à la vie populaire c'est avant tout pour la force tragique qu'elle recèle et dans laquelle il retrouve les passions shakespeariennes :

[...] chez Shakespeare, Eschyle, Tolstoï, Dostoïevski et les autres, il se passait des drames et des conflits terribles et j'ai découvert dans les Caraïbes que dans la vie dans laquelle j'avais été élevé et dans laquelle vivaient tous ces professeurs, il n'y avait rien qui correspondait aux conflits violents, aux explosions et aux événements marquants et intéressants que je trouvais dans la littérature classique, de sorte que je parlais [aux gens ordinaires] parce qu'ils m'exprimaient et me racontaient des choses que je lisais. Ma famille ne sortait pas d'un cercle social très restreint, ils devaient maintenir leur distance du fait de leur statut d'enseignant (James C. L. R., et al., $1980: 3)^{21}$.

21 Ce lien avec le tragique est particulièrement intéressant en ce sens que dans ses écrits historiques, et particulièrement avec Les Jacobins Noirs, James élève l'histoire à la hauteur de la tragédie, comme si la vieille Europe n'était plus capable de produire des Othello et que seul Haïti avait encore cette capacité (Cudjoe S. R., et al., 1992: 128).

Alfred Mendes déclara en 1972 :

James et moi-même nous nous éloignâmes des normes par la sélection de nos sujets, par nos choix de vie, par l'utilisation d'une langue nouvelle. Et ces choix sont toujours ceux de nos successeurs caribéens. Nous avons créé les cadres qui sont toujours utilisés de nos jours. Et ce sont ces postulats qui ont permis aux romanciers antillais d'exister.

(King, B.A., $1979: 57)^{22}$

Cependant ces nouvelles formes ne sont pas exemptes d'une forme d'imitation, à commencer avec James par celle de ses romanciers préférés, Thackeray et Bennett, 
comme le remarque Anna Grimshaw dans son introduction au C. L. R. James Reader (James C. L. R., et al., $1992: 4-5)^{23}$. Mais cette imitation stylistique tourne parfois à la parodie, comme dans ces passages où le fil de la narration et des dialogues est interrompu sans cesse par la multiplication des interférences, parenthèses et prises de parole impromptues. En voici un parmi d'autres, dans lequel la parenthèse inclut étrangement la suite du dialogue :

Elle lui a dit aussi facilement que le bonjour qu'elle était fiancée pour être mariée à un médecin, qui l'avait séduite puis abandonnée (mais Ella répondit dit non : elle avait été sauvage dès le début, et Sonny n'était pas son premier né).

(James C. L. R., $1936: 49)^{24}$

A propos de ces exercices de style et ces passages écrits «à la manière de », nous pouvons faire référence à Homi Bhabha qui écrit qu'il faut interpréter les cultures subalternes en ce qu'elles sont caractérisées par ce qu'il rassemble sous l'expression de " colonial mimicry», mimétisme, ou imitation coloniale (Bhabha H., $1984: 130)^{25}$. En ce sens James utilise non sans ironie les leçons d'ironie qu'il a trouvées chez Thackeray.

James pense en termes de style, et d'esthétique d'abord en référence au cricket, où tout est style, depuis la pratique du sport en lui-même, jusqu'aux articles sportifs qu'il lit avec avidité, d'autant plus qu'il écrit lui-même pour la presse sportive. Son modèle incontesté est le journaliste britannique Neville Cardus (1888-1975) et son style fait de métaphores (Bateman A., 2009).

Par cette ambition à fonder un style propre, Minty Alley peut aussi être interprété comme une entreprise au service de la dignité des colonisés, un plaidoyer pour l'égale dignité des Trinidadiens de toutes origines et de toutes couleurs confondues, dans la tradition de la "dignité de la race », de la littérature de défense de la race, « vindication of race ", comme le fait l'historien et exécuteur testamentaire de James Robert A. Hill (1999). Cependant il nous semble que la vision que donne James des inégalités ethnoraciales est plus complexe.

\section{Être ou ne pas être créole}

Le thème des différences de couleur, d'origine est central dans le roman, qui ne comporte aucun personnage blanc. La mère du héros, Haynes, lui déclare : «Mon fils tu es noir, [et] dans ces petites îles pour qu'un Noir soit indépendant il a besoin d'avoir de l'argent ou une profession $»^{26}$. La complexité du microcosme de la cour $n^{\circ} 2$ résume la société de l'île, en termes de conditions sociales, de nuances de couleurs de peau et d'origine diverses. Enfant d'un couple d'enseignants, James a appris à toujours porter sa respectabilité sociale comme une "armure " (Harris L., $2018: 21$ ). Cependant son roman, comme d'ailleurs sa nouvelle Triumph, donne à voir une image de la vie locale que la petite bourgeoisie respectable préférait ignorer. Un critique désigne le courant littéraire de la barrack-yard literature comme "des fictions anti-establishment" (Rodriques J., 2019 : 231). Les religions locales y sont présentées, comme la foi Obeah, un système religieux d'origine africaine fondé sur des pratiques individuelles de guérison spirituelle dont le premier témoignage connu provient de la Jamaïque au XVIII siècle. Ces croyances n'étaient évidemment pas bien perçues et considérées comme des formes de sorcellerie primitive (Rodriques J., 2019). Ainsi Mrs Rouse chasse Philamen, sur les conseils d'un Obeah, un guide spirituel, car celle-ci est d'origine indienne, une coolie selon le terme local : 
Vous voyez, M. Haynes, j'ai quelqu'un qui me guide dans ma vie. C'est un homme qui peut faire des choses et je peux vous dire qu'il m'a beaucoup aidée. Et quand je suis allée le voir une fois, il m'a dit que mon le sang et le sang de coolie ne prennent pas. Il dit que c'est pour cela que M. Benoit me traite comme il l'a fait

(James C. L. R., 1936 : 240).

Dans l'anglais des Caraïbes, «le sang prend ou ne prend pas » quelqu'un si on est attiré ou au contraire repoussé par l'autre. L'homme qu'elle aime, Benoit, est un métis, un Noir avec des origines indiennes, ce que les habitants de Trinidad comme de Guyana nomment avec mépris un "dougla», une catégorie hybride le plus souvent ignorée (Ibid., p. 30). Benoit est d'ailleurs un personnage au moins aussi important que Haynes (Mehta B., 2002). Finalement Mrs Rouse va rapidement regretter d'avoir chassé sa fidèle servante. James dénonce le ridicule des stéréotypes raciaux et plaide ainsi pour une meilleure entente entre les Trinidadiens d'origine indienne, représentés par Philamen, et les Noirs. Il ne cherche pas à délimiter une identité trinidadienne, mais la représente dans l'hybridité, le mouvement. Ainsi que l'écrit Stuart Hall : «ce qui est unique avec [la société caribéenne] c'est que la moitié d'entre elle appartient [à une autre culture] (Hall S., $1992: 5)^{27}$.

Ce qui n'empêche nullement James de revendiquer en même temps l'identité d'abord britannique des Trinidadiens, mais principalement sur le plan politique. En effet en parallèle de ses écrits littéraires, il entame une biographie, The Life of Captain Cipriani, sur le dirigeant de la Trinidad Workingmen's Association, la TWA, le principal syndicat de l'île formé en 1898. Arthur Andrew Cipriani (1875-1945), un Créole français d'origine corse et ex-capitaine durant la guerre, en devient en 1923 le leader (Samaroo B., et al., 1972). Dès les premières pages James écrit :

Ce que celui qui ignore tout de ces îles doit planter fermement dans son esprit, c'est que ces peuples ne sont pas des sauvages, qu'ils ne parlent que la langue anglaise, (enfin pas exclusivement), qu'ils n'ont pas d'autre religion que le christianisme (encore une fois pas uniquement), et qu'en réalité toute leur vision du monde est celle de la civilisation occidentale, modifiée et adaptée aux circonstances particulières de leur vie.

(James C. L. R., $2014: 10)^{28}$

Ce texte n'est pas la recherche d'une quelconque identité, mais revendique la reconnaissance du fait que les Trinidadiens sont eux aussi « civilisés ». Sa vision d'une identité caribéenne est par nature non essentialiste, en mouvement, et d'une certaine façon elle annonce le concept d'hybridité développé par Paul Gilroy, que Gilroy illustra précisément avec l'exemple des écrits de James (Gilroy P., 1993 : 159).

31 James n'a encore cependant que peu d'intérêt pour la politique. Sa première intervention strictement politique ne date que de 1931 lorsqu'il répond à un texte ouvertement raciste du botaniste Sidney Harland (1891-1982). Celui-ci cherchait à démontrer l' " infériorité mentale des Noirs » (James C. L. R., 2014 : 17). Il n'est pas alors engagé politiquement. L'amour de la littérature et du cricket l'ont fait vivre tel un exilé britannique dans la Caraïbe, et le personnage de Haynes exprime ce sentiment d'aliénation. La littérature est alors le mode par lequel il comprend la société et l'histoire comme il le déclare à ses étudiants :

Pour étudier un sujet historique, il faut avoir une idée de la situation économique, une idée de la situation politique et une connaissance de la situation littéraire. Ce n'est que lorsque vous connaissez ces trois éléments que vous avez une idée de l'évolution historique de la période.

(Berman P. et al., $1981: 13)^{29}$ 
Son amour des lettres et du cricket a fini par le rapprocher de son île. Il la quitte cependant en 1932. Son ami Constantine s'est installé en 1929 dans le Lancashire, où il a été embauché par le club de Nelson et en 1932 James l'y rejoint pour lui prêter sa plume et terminer la rédaction des mémoires du sportif, intitulées Cricket and I (Constantine L., 1933). Il emporte aussi avec lui un premier écrit plus directement politique, The Life of Captain Cipriani. Son ambition est de rejoindre à Londres le groupe de Bloomsbury autour des époux Woolf.

\section{Trinidad, creuset d'une vision politique globale}

Trinidad est devenue une colonie de la Couronne en 1832, ce qui signifie que le gouverneur nommé à Londres détient l'ensemble des pouvoirs, y compris, jusqu'en 1925, celui de sélectionner les membres qui participent au conseil législatif local. La population est alors constituée d'environ $4 \%$ de Blancs, $15 \%$ de Créoles et $80 \%$ dits « coloured" dont la moitié environ d'origine indienne et l'autre moitié d'ascendance africaine. Le retour des soldats mobilisés durant la Première Guerre mondiale alimente le mécontentement social. Durant la guerre, massivement mobilisés, les Trinidadiens ont été maltraités par leurs officiers ${ }^{30}$. En Italie certains se sont mutinés. Cet état d'esprit se prolonge ensuite lors de la grève quasi générale de 1919, que le gouverneur réprime avec l'aide de volontaires blancs membres notamment du Union $\mathrm{Club}^{31}$. James se défend d'être impliqué directement dans la TWA. Il déclare dans une interview à Paul Buhle que Cipriani ne l'a jamais encouragé à le rejoindre, du fait de son emploi de fonctionnaire, qui lui interdisait tout engagement. Cependant, dans une autre interview il dit aussi que Cipriani venait sans cesse lui demander conseil sur tel ou tel problème (James C. L. R., 1992 : 60). Par ailleurs, il écrit pour le journal du syndicat, le Labour Leader, mais exclusivement dans la rubrique sportive - ces articles ne sont pas signés en raison de son emploi de fonctionnaire qui lui interdit de prendre ainsi parti. Nous présentons ci-dessous une couverture du journal de la TWA, le Labour Leader, en 1928, avec les résultats des élections à Port-of-Spain. Remarquons que la mention dans la manchette du «seul journal politique de l'île» contient un double sens: d'abord un sens littéral, pour affirmer que les autres journaux ne sont pas engagés de cette façon. Mais il évoque aussi l'évolution du statut de la colonie, alors que Londres autorise pour la première fois des élections et donc plus largement une vie politique libre. 


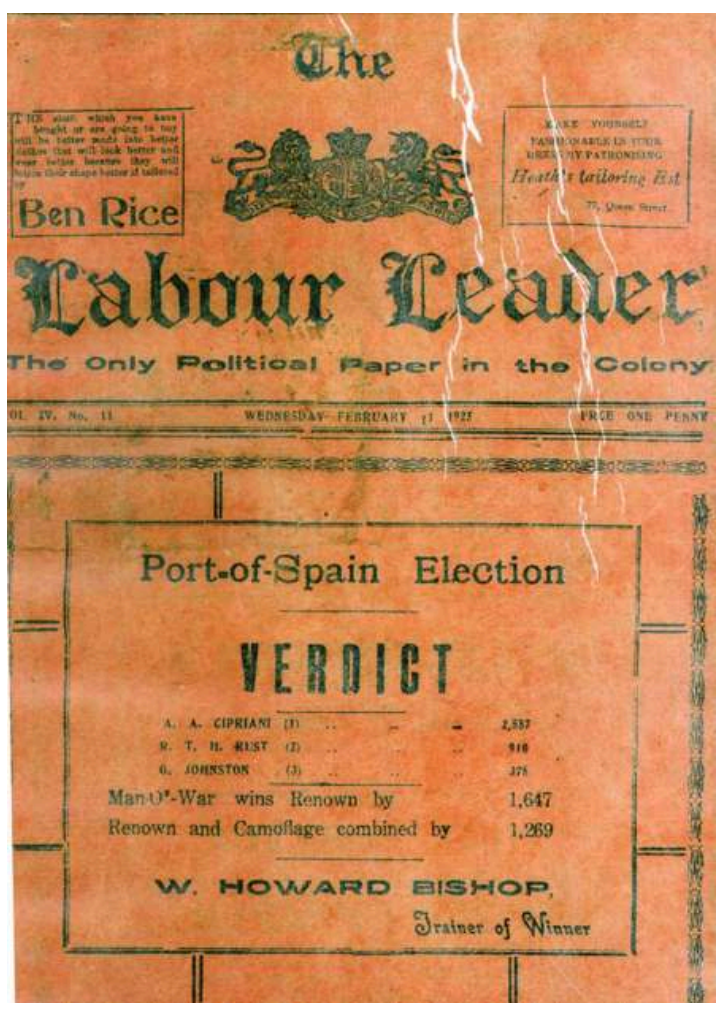

Figure : Première de couverture du Labour Leader en février 1928. Cipriani est élu maire.

Source : Trinidad Ministry of National Diversity and Social Integration http://natt.gov.tt/sites/default/ files/pdfs/Road\%20to\%20Nationhood-Storyboards-2014-2_Part1.pdf

James entreprend donc en 1929 la rédaction d'une biographie de Cipriani, autour de son combat politique lors des premières élections du Legislative Council en $1925^{32}$. Il s'agit comme il l'annonce dès les premières lignes d'une réflexion sur la nature du gouvernement de la Couronne. Il choisit pour la première fois la forme de la biographie, et c'est un genre qu'il utilisera ensuite avec la vie de Toussaint-Louverture à la fois avec les Jacobins Noirs et le drame Toussaint Louverture (James C. L. R., 1934 ; 1938). Cela lui permet de questionner la relation entre le leader et le peuple, entre le parti et les masses, ou en termes marxistes, comme il le formulera ensuite, le rôle de l'individu dans l'histoire face à la permanence des infrastructures (James C. L. R., 2009; 2000).

La biographie de Cipriani sert de prétexte à un plaidoyer politique. James écrit que Cipriani lui a fait découvrir qu'il était aussi caribéen. Les premières pages présentent successivement les différentes catégories de la population selon leur identité raciale, dans un raisonnement qui essentialise les qualités propres aux uns et aux autres. La population de couleur y est considérée comme un tout homogène, et le seul paragraphe à propos des Indo-Trinidadiens affirme qu'il n'y a "pas le moindre conflit » entre eux et les Noirs (James C. L. R., $2014: 57)^{33}$. On sait pourtant que la vie politique de l'île est déjà marquée par ces tensions, qui vont aller croissant. Mais James veut démontrer avant tout qu'il y a suffisamment de gens éduqués à Trinidad pour assumer pleinement l'indépendance. Il insiste d'ailleurs sur le fait que le peuple de Trinidad n'a plus de liens ni avec l'Afrique, ni avec l'Inde, ce qui évitera tout conflit entre « les idées récemment assimilées de la démocratie moderne, et les anciennes traditions tribales ou liées aux castes" (Ibid.: 16) ${ }^{34}$. Si The Life est un texte précurseur du combat pour la décolonisation, celle-ci doit se réaliser au nom du fait que les Trinidadiens sont avant tout des Britanniques sous les tropiques. D'ailleurs il ne revendique alors que 
l'évolution constitutionnelle vers le self-government, qui n'est pas encore une indépendance pleine et entière. En 1932 en Angleterre, à la demande de son éditeur, il reprend des extraits de The Life qui ne portent pas sur Cipriani et les publie sous la forme d'une brochure politique The Case for West-Indian Self Government (1933) ${ }^{35}$. Son cheminement politique vers l'anticolonialisme en est encore à ses premiers pas. A Nelson il découvre le marxisme et évolue rapidement vers un engagement politique plus radical, du cricket au marxisme.

\section{Cricket et marxisme shakespearien}

Les enjeux politiques traversent le cricket et James écrit que «[Celui-ci] m'a plongé dans la politique bien avant que j'en fusse conscient. Lorsque je me suis intéressé à la politique je n'avais plus grand-chose à découvrir » (Ibid. : 17-18) ${ }^{36}$. Les clubs de cricket suivent strictement les lignes de couleur et les délimitations sociales. Le club de James, le Shannon, était celui des classes moyennes inférieures noires (Nielsen, 1997, 180). Il suit de près toutes les performances des clubs britanniques mais aussi dans tout l'empire, à commencer par les Caraïbes. Cette dimension internationale, impériale plutôt, ne cesse de poser la question coloniale, à commencer du fait du départ des meilleurs joueurs vers l'Angleterre. Dans un court texte, l'historien E. P. Thompson observe à propos de James que « les théoriciens américains ne [le] comprendront pas, mais la clé de tout réside dans son appréciation du cricket » (Thompson, 1981) ${ }^{37}$. En 1975 James publiera un ouvrage de 500 pages sur le cricket et la manière dont la presse essentialise les sportifs noirs (James C. L. R., 1975). Sa compréhension des enjeux coloniaux mis en œuvre par ce sport l'a préparé à la découverte du marxisme.

Celle-ci est d'abord purement intellectuelle (James C. L. R., 2000 : 68). Entre 1933 et 1934 il lit Trotsky :

J'ai lu l'Histoire de la révolution russe parce que j'étais très intéressé par cet événement et ce livre semblait offrir une analyse de la société moderne (Trotsky, 1979, [1932]). Après l'avoir terminé, au printemps 1934, j'étais devenu trotskyste en théorie, et j'ai rejoint le mouvement par la suite. Il était clair pour moi que je n'allais pas devenir un stalinien (Berman P. et al., 1981, p. 17) ${ }^{38}$.

Il faut noter que le style de Trotsky historien lui semble essentiel: «Il y a là une profonde leçon non seulement d'histoire mais aussi d'esthétique » (James et al., 1994 : 123). Il retiendra la leçon pour les Jacobins Noirs dans lequel la frontière entre l'œuvre littéraire et scientifique s'efface partiellement (Marriott D., 2015). Il rejoint l'Independant Labour Party, un parti socialiste formé dès 1893 qui conserva son indépendance hors du Parti travailliste formé en 1900, au sein duquel agit le courant trotskyste.

\section{Conclusion}

Le parcours de James tel que nous l'avons décrit pourrait donner une fausse impression de simplicité autour d'un basculement d'une éducation victorienne à Trinidad qui s'accompagne de ses essais littéraires, vers le marxisme et les écrits historiques en Angleterre $^{39}$. Ce schéma sans doute trop simple suit ce que l'historien Dipesh Chakrabarty nomme les lignes de l'historicisme, «d'abord en Occident, ailleurs ensuite" avec pour James un double décalage temporel et spatial, puisqu'il est à Trinidad un homme de l' « ère victorienne avec une graine de révolte » (James C. L. R., 
2000 : 192 ; Chakrabarty D., $2000: 4)^{40}$. Si James illustre par ses déplacements les liens entre les marges colonisées et le centre impérial, sa pensée en mouvement refuse l'essentialisation d'un sujet colonial et permet de penser l'hybridation : elle s'incarne dans le cricket, un sport d'origine britannique dont les colonisés se sont emparés, tout comme dans ses écrits littéraires lorsqu'il créolise Othello sous les traits tragique de Toussaint Louverture. Il est sans doute possible d'interroger dans ses écrits, comme l'écrit Marie Cuillerai, les "processus de dislocation, traduction, l'imitation qui hybrident et négocient, autrement dit réagencent la symétrie identité à soi / altérité » (Cuillerai M., 2010).

Par la multiplicité des liens souvent inattendus que James établit, entre révolution et esthétique tragique, entre histoire et style littéraire, entre culture populaire et anticolonialisme, il déjoue sans cesse le paradigme colonial dénoncé par Homi Bhabha. Il nous fournit des outils pour penser la discontinuité, et refuser une lecture du centre à la marge, ou des marges au centre, car donner la primauté aux dominés ne permet pas de sortir du paradigme colonial. Thomas Brisson a récemment écrit à propos d'un « lien contre-intuitif entre occidentalisation et critique de l'Occident " et James semble illustrer cette idée, lui qui mit sa culture classique au service d'une pensée décentrée (Brisson T., $2018: 8$ ).

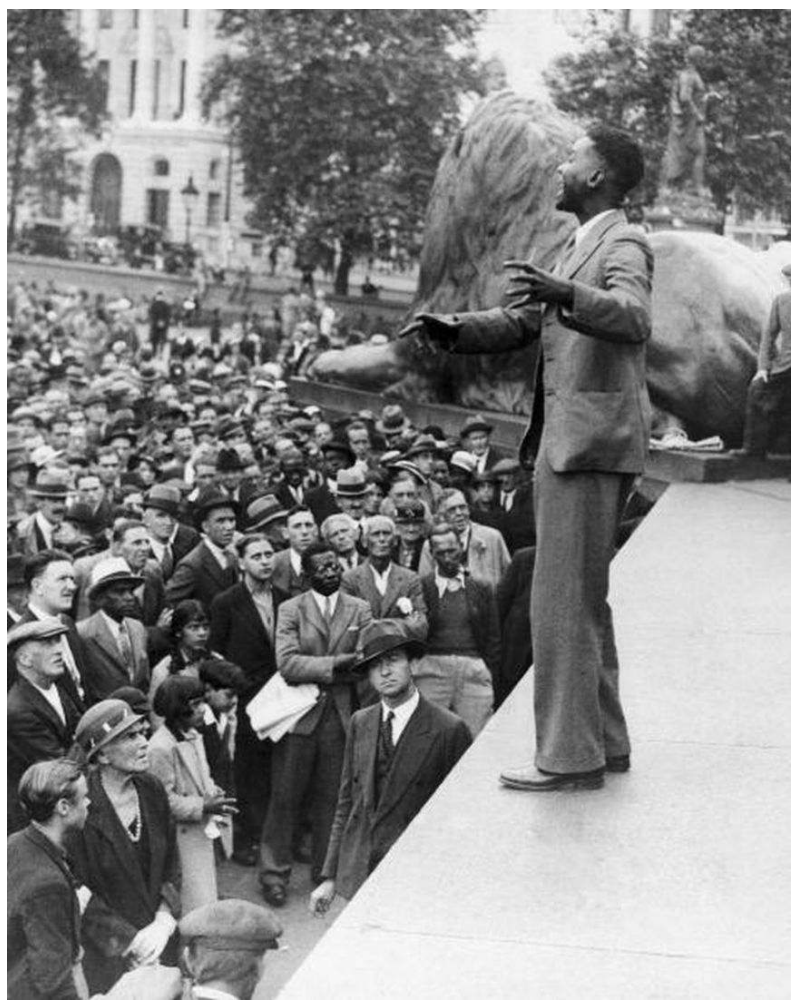

Figure : C. L. R. James au « Speaker's Corner », à Trafalgar square, en 1935.

Source : https://uwimuseum.wordpress.com/2017/01/10/check-out-girvan-on-clr-james/speech-of-aman-against-the-embargo-in-ethiopia-at-trafalgar-square-in-1935/ page consultée le 24 septembre 2020. 


\section{BIBLIOGRAPHIE}

Agard, John, « Prospero Caliban Cricket », The Massachusetts Review, 1994, vol. 35 n 3-4. p. 546-548.

Bateman, Anthony, «"From Far It Look Like Politics”: C.L.R. James and the Canon of English Cricket Literature ", Sport in Society, 1 mai 2009, vol. 12 n 4-5, p. 496-508.

Berman, Paul et Buhle, Paul, C.L.R. James: His Life and Work, Chicago, Sojourner Truth Organization, 1981.

Bhabha, Homi, « Of Mimicry and Man: The Ambivalence of Colonial Discourse », October, vol. 28,1984, p. 125.

Bhabha, Homi K., Les lieux de la culture une théorie postcoloniale, Paris, Payot, 2007.

Bogues, Anthony, Caliban's Freedom: the Early Political Thought of C. L. R. James, Londres : Pluto Press, 1997.

de Boissière, Ralph, « The Rise of the Beacon Group: extract from Ralph de Boissière's (as yet unpublished) autobiography ", Journal of West Indian Literature, vol. 9 n 2, 2001, p. 8-17.

Booker, M. Keith, Encyclopedia of Literature and Politics: Censorship, Revolution, and Writing, Westport (Conn.), Greenwood Press, 2005.

Brisson, Thomas, Décentrer l'Occident : les intellectuels postcoloniaux chinois, arabes et indiens et la critique de la modernité, Paris, La Découverte, 2018.

Buhle, Paul, « Review of C. L. R. James and Creolization: Circles of Influence », African American Review, vol.37 nº 2 / 3, 2003, p. 467-468.

Buhle, Paul, C. L. R. James the Artist As Revolutionary, London, New York, Verso, 1988.

Carby, Hazel V., « Proletarian or Revolutionary Literature: C. L. R. James and the Politics of the Trinidadian Renaissance ", New Formations, vol 10, Radical Differences, 1990 (Lawrence \& Wishart). En ligne : https://www.lwbooks.co.uk/new-formations/10/proletarian-orrevolutionary-literature [consulté le 30 juin 2020].

Caribbean Artists Movement, Savacou, Kingston, Jamaica, Caribbean Artists Movement, vol 2, 1970.

Chakrabarty, Dipesh, Provincializing Europe: Postcolonial Thought and Historical Difference. Princeton, Oxford, Princeton university press, 2000.

Chivallon, Christine, « Black Atlantic Revisited. Une relecture de Paul Gilroy pour quelques prolongements vers le jazz ", L'Homme. Revue française d'anthropologie, 3 octobre $2008 \mathrm{n}^{\circ} 187-188$, p. 343-374.

Constantine, Learie Nicholas, Cricket and I, London, P. Allan, 1933.

Cudjoe, Selwyn R., Anna Grimshaw, Paget Henry, et al., « C. L. R. James Misbound », Transition Transition. $1992 \mathrm{n}^{\circ}$ 58. p. 124.

Cuillerai, Marie, « Le Tiers-espace : une pensée de l'émancipation? », Acta Fabula, vol 11, n 125, janvier 2010. En ligne : https://www.fabula.org:443/revue/document5451.php [consulté le 29 juin 2020]. 
Cynthia Hamilton, «A Way of Seeing: Culture as Political Expression in the Works of C. L. R. James », Journal of Black Studies, vol 22 n³, 1992, p. 429-443.

Du Bois, William Edward Burghardt, Les âmes du peuple noir [1903], traduction de Magali Bessone, Paris, La Découverte, 2007.

Gilroy, Paul, The Black Atlantic: Modernity and Double Consciousness, Londres, Verso. 1993.

Hall, Stuart, « C. L. R. James: A Portrait » C.L.R. James's Caribbean. Durham NC, Duke University Press, 1992.

Hall, Stuart et James C. L. R., « Conversation with C.L.R. James » Rethinking C.L.R. James: A Critical Reader, Cambridge, Mass., Blackwell, 1995, p. 18.

Harris, Laura, « What Happened to the Motley Crew?: James, Oiticica, and the Aesthetic Sociality of Blackness » Experiments in Exile, $1^{\text {re }}$ éd. New York, Fordham University Press. 2018, p. 17-60. (C. L. R. James, Hélio Oiticica, and the Aesthetic Sociality of Blackness). En ligne : https:// www.jstor.org/stable/j.ctv8bt1hd.4 [consulté le 27 juin 2020].

Henry, Paget et M. Buhle Paul, C. L. R. James's Caribbean, Durham, N.C., Duke University Press, 1992. Hill, Robert A., « C. L. R. James: The Myth of Western Civilization » Enterprise of the Indies, Port-ofSpain, Trinidad and Tobago Inst. of the West Indies, 1999.

Høgsbjerg, Christian, C. L. R. James in Imperial Britain, Durham NC, Duke University Press, 2014. Høgsbjerg, Christian, «"A Thorn in the Side of Great Britain": C. L. R. James and the Caribbean Labour Rebellions of the 1930's ", Small Axe, vol 15 n $^{\circ} 2,27$ juillet 2011, p. 24-42.

James, C. L. R., Toussaint Louverture: The Story of the Only Successful Slave Revolt in History; A Play in Three Acts, Durham, N.C., Duke University Press, 2012.

James, C. L. R. The Black Jacobins: Toussaint L'ouverture and the San Domingo Revolution, Londres, Penguin UK [1938], 2001a

James, C. L. R., « La Diablesse », Journal of West Indian Literature, vol 9 n² 2, p. 4-7. $2001 \mathrm{~b}$.

James, C. L. R., « Lectures on The Black Jacobins ", Small Axe: A Caribbean Journal of Criticism, $\mathrm{n}^{\circ} 8$, septembre 2000, p. 65.

James, C. L. R., Beyond a Boundary, London : Serpent's Tail, 2000.

James, C. L. R., A Majestic Innings: Writings on Cricket, London, Allison \& Busby, 1986 [1975].

James, C. L. R., Marxism and the Intellectuals, 1962. En ligne : https://www.marxists.org/archive/ james-clr/works/1962/destruction-paper/index.htm, [consulté le 27 juin 2020].

James, C. L. R., Minty Alley; A Novel, London, Secker \& Warburg, 1936.

James, C. L. R. et Anna Grimshaw, C. L. R. James Reader, Oxford ; Cambridge, Mass, Blackwell Publishers, 1992.

James, C. L. R, McLemee Scott, Le Blanc Paul, et al., C.L.R. James and revolutionary Marxism: selected writings of C.L.R. James, 1939-1949, Chicago, Haymarket Books, 1994.

James, C. L. R et Ramchand, Ken, « Interview with C. L. R. James », 1980, http://www.clrjames.uk/ wp-content/uploads/2015/10/Interviews-with-C.L.R.-James-by-Ken-Ramchand.pdf, [consulté le 24 septembre 2020].

James, C. L. R. "The Making of a Literary Life: C. L. R. James interviewed by Paul Buhle », C. L. R. James's Caribbean. Paget Henry; Paul Buhle (eds). 1992, p. 56-62. 
James, C. L. R., World revolution, 1917-1936: The Rise and Fall of the Communist International, London, Secker \& Warburg, 1937.

James, C. L. R., The life of Captain Cipriani: an account of British government in the West Indies, with the pamphlet The case for West-Indian Self Government, Durham, Duke University Press, 2014 [Nelson, Lancs., Coulton, 1932].

James, C. L. R., «The Black Scholar Interviews: C. L. R. James ", Black Scholar, vol $42 \mathrm{n}^{\circ}$ 2, Summer 2012, p. 22-30.

James, C. L. R., You Don't Play with Revolution: The Montréal Lectures of C. L. R. James, Oakland, AK

Press. 2009.

James, C. L. R., At the Rendezvous of Victory: Selected Writings. London, Allison \& Busby, 1984.

Joubert, Claire, "Théorie en traduction : Homi Bhabha et l'intervention postcoloniale ", Littérature, $\mathrm{n}^{\circ} 154 \mathrm{n}^{\circ} 2,2009$, p. 149-174.

Kamugisha, Aaron, « C. L. R. James's Twentieth Century Literary Journeys » The Routledge companion to Anglophone Caribbean literature, Londres, Routledge, 2014.

King, Bruce Alvin, West Indian Literature, London, Basingstoke : Macmillan International college, 1979.

King, Nicole, C. L. R. James and Creolization: Circles of Influence, Jackson, University Press Of Mississippi, 2007.

Lai, Walton Look, Buhle, Paul M et Paget, Henry, « C. L. R. James and Trinidadian Nationalism », dans C. L. R. James's Caribbean, Durham, N.C, Duke University Press, 1992, p. 174-209, https:// www.academia.edu/23279823/C._L._R._James_and_Trinidadian_NationalismC. L. R. James and Trinidadian Nationalism [consulté le 26 avril 2019]

Marriott, David, « The ocular truth: C. L. R. James's England », Critical Quarterly, vol 57 n 3, 2015, p. 35-50.

Mehta, Brinda, « Addressing Marginality Through the "Coolie/Dougla" Stereotype in C. L. R. James's Minty Alley », The CLR James Journal, 2002, vol 9 n 1, p. 37-66.

Mendes, Alfred H., « Boodhoo », The Beacon: A Monthly Review, 1 n 12, Avril 1932.

Nielsen, Aldon Lynn, C. L. R. James: A Critical Introduction, Jackson, University Press of Mississippi, 1997.

Parris, Elliot D., « Minty Alley » in Berman, Paul, Buhle, Paul, et sojourner Truth organization (eds.), C. L. R. James: his Life and Work, Chicago, Sojourner Truth Organization, p. 97, Urgent Task, $\mathrm{n}^{\circ} 12,1981$.

Renault, Matthieu, C. L. R. James: la vie révolutionnaire d'un "Platon noir", Paris, La Découverte, 2016. Rodriques, Janelle, Narratives of Obeah in West Indian literature: moving through the margins. Londres : Routledge, 2019, en ligne : https://search.ebscohost.com/login.aspx?

direct=true\&scope=site\&db=nlebk\&db=nlabk\&AN=2097776 [consulté le 26 juin 2020].

Rosengarten, Frank, Urbane Revolutionary: C. L. R. James and the Struggle for a New Society, Jackson, University Press of Mississippi, 2007.

Rush, Anne Spry, Bonds of Empire: West Indians and Britishness from Victoria to Decolonization, Oxford, Oxford University Press, 2011. 
samaroo, Brinsley et Girvan, Cherita, « The Trinidad Workingmen's Association and the Origins of Popular Protest in a Crown Colony ", Social and Economic Studies, vol $21 \mathrm{n}^{\circ}$ 2, 1972, p. 205-222.

sander, Reinhard W, From Trinidad: an Anthology of Early West Indian Writing, New York, Holmes and Meier Publishers, 1978.

sander, Reinhard W. et Munro, Ian, Kas-kas, interviews with three Caribbean writers in Texas: George Lamming, C. L. R. James, Wilson Harris, Austin, African and Afro-American research Institute, University of Texas at Austin, 1972.

Thackeray, William Makepeace, Vanity Fair: A Novel without a Hero, Oxford, Oxford University Press, 2015 [1847-1848].

Thompson, Edward Palmer, «C. L. R. James at 80 », in Berman, Paul, Buhle, Paul et sojourner Truth organization (eds.), C. L. R. James: His Life and Work, Chicago, Sojourner Truth Organization, Urgent Task, n 12, 1981.

Trotsky, Léon, Histoire de la révolution russe, Paris, éd. du Seuil, 1979 [1930].

worcester, Kent, C. L. R. James: a Political Biography, Albany, SUNY Press, 1996.

wynter, Sylvia, «Beyond the Categories of the Master Conception: the Counterdoctrine of the Jamesian Poiesis » C. L. R. James's Caribbean, Durham, NC, Duke University Press, 1992, p. 63-91.

\section{NOTES}

1. Dans une récente biographie de C. L. R. James, Matthieu Renault débute son récit en soulignant précisément ces déplacements incessants (Renault M., $2016: 8$ ).

2. Un des rares ouvrages sur la dimension caribéenne de James est celui de Nicole King. (2007)

3. " a Negro yet British, colonial native yet culturally part of the public school code, attached to the cause of the proletariat yet a member of the middle class, a Marxian yet a Puritan, an intellectual who plays cricket, of African descent yet Western, a Trotskyist and Pan-Africanist, a Marxist yet a supporter of black studies, a West Indian majority black yeti an American minority black».

4. « Ni souvenirs sur le cricket, ni autobiographie » écrit-il dans sa préface. Par ailleurs il a donné de nombreuses interviews. (James C. L. R., et al., 1980 ; James, C. L. R., 2012)

5. «Cricket is a game of high and difficult technique. If it were not it could not carry the load of social response and implications which it carries ».

6. «The class and race rivalries were too intense. They could be fought out without violence or much lost except pride and honour. Thus, the cricket field was a stage on which selected individuals played representative roles which were charged with social significance ».

7. « I really had an excellent education to prepare me for being a British intellectual ».

8. Trinidad fut à partir de 1498 une colonie espagnole, La monarchie Bourbonne fit venir à la fin $\mathrm{du}$ XVIII ${ }^{\mathrm{e}}$ de nombreux colons français, pour acclimater dans l'île la culture du coton et du sucre. En 1797 Trinidad est conquise par les Britanniques et connaît un afflux d'esclaves sans précédent. Après l'abolition de l'esclavage, en 1838, les planteurs de l'île ont recours aux travailleurs sous contrat originaires le plus souvent d'Inde. Du fait de cette histoire Trinidad n'a connu l'esclavage que durant une période relativement brève, et se distingue aussi des îles voisines par le nombre important de Noirs libres avant cette date.

9. Ce père se révèle être un excellent «cutter »-du nom de cette technique au cricket. Les termes qui désignent différentes techniques, types et phases de jeu au cricket, comme «Bowler», « 
swinger » et "cutter » demeurent malheureusement intraduisibles, et contribuent à rendre les règles du cricket impénétrables au commun des mortels hors de la sphère impériale britannique. 10. « had revolted against the revolting contrast between his first-class status as a cricketer and his third-class status as a man ... The restraints imposed upon him by social conditions in the West Indies had become intolerable and he decided to stand them no longer ".

11. Comme le signale cet article d'une encyclopédie littéraire, James, du fait de la largeur et de la diversité de ses vues, était parfois surnommé le Platon noir de son vivant. Ce surnom fut repris par le journal The Times en 1980. (Booker, M. K., 2005 : 378 ; Henry P. et al., 1992 : 5)

12. Il lit indistinctement Dickens, Thackeray, Walter Scott, et Herman Melville. Mais surtout il lit et relit plus de vingt fois la Foire aux Vanités (Thackeray W. M., 1847-48).

13. "I laughed without satiety at Thackeray's constant jokes and sneers and gibes at the aristocracy and at people in high places. Thackeray, not Marx bears the heaviest responsibility for me ".

14. Auparavant ce cercle d'amis se dénommait le Maverick.

15. La première, Divina Pastora, rencontre le succès et est éditée en Angleterre en 1927 (James C. L. R., et al., 1992)

16. Trinidad est publiée une première fois en Décembre 1930, puis une seconde en avril 1931 (Sander, 1978 : 3). Leur cercle réunit notamment Alfred H. Mendes (1897-1991) et Ralph de Boissière.

17. «A movement of enlightenment » (Bogues A., 1997 : 20)

18. Ces nouvelles publiées en 1931 ont été republiées : voir 2001b)

19. " as if by accident ".

20. Voici l'extrait de son interview : «En ce qui concerne le contenu conceptuel de la littérature, le groupe [The Beacon] est resté conservateur. Pour la plupart d'entre nous, écrire sur les mœurs des pauvres dans les cours de baraques était une évidence. Nous n'en sommes pas arrivés à faire des travailleurs les héros de leur lutte contre l'employeur. La possibilité existait, mais nous étions trop aveuglés par notre éducation pour le percevoir et le concevoir, et même si nous avions perçu cette possibilité les canons littéraires que nous avons acceptés nous auraient empêchés de lui donner une expression». ("Where the idea-content of literature was concerned the group remained conservative. To write of the mores of the poor in barrack yards was as far as most of us went. We did not reach the stage of making workers the heroes in their struggle against the employer. The possibility existed, but we were too blinded by our education and upbringing to perceive it and give it rein, and even had we perceived that possibility] the literary canons we accepted would have prevented us giving it expression ».

21. "But in Shakespeare, Aeschylus, in Tolstoy, in Dostoyevsky and the rest of them, things were taking place and tremendous conflicts were taking place and I found in the Caribbean, that in the life in which I had been brought up and in which all those teachers lived there was nothing corresponding to the violent conflicts and explosions and peculiar and interesting happenings that I found in Classic Literature, so that I talked to them because they were expressing and telling me about things that I was reading about. My family kept within a very narrow range, they had to be, being teachers ".

22. Mendes was quoted as saying in 1972: "James and I departed from the convention in the selection of our material, in the choice of a strange way of life, in the use of a new dialect. And these departures are still with our Caribbean successors. We created the pattern that is still in use. And these are the postulates that brought West Indian novelists into being ".

23. Il s'agit du romancier anglais Enoch Arnold Bennett (1867 - 1931).

24. "She told him as easily as good morning that she had been engaged to be married to a doctor, who had seduced her and then deserted her. (But Ella said no: she had been wild from early, and Sonny was not her firstborn.) ». 
25. «[...] alors le mimétisme colonial est le désir d'un Autre réformé, reconnaissable, comme sujet d'une différence qui est presque la même, mais pas tout à fait. L'effet du mimétisme sur l'autorité du discours colonial est profond et inquiétant ». (" If I may adapt Samuel Weber's formulation of the marginalizing vision of castration, then colonial mimicry is the desire for a reformed, recognizable Other, as a subject of a difference that is almost the same, but not quite. The effect of mimicry on the authority of colonial discourse is profound and disturbing " (Homi Bhabha, 1984). Il faut noter que les concepts souvent rassemblés sous l'expression post-coloniale semblent illustrer par eux même les enjeux de la traduction comme le souligne l'article de Joubert sur la question de la traduction de mimicry (Joubert C., 2009, p. 3).

26. (James C. L. R., 1936, p. 22) «You are black my boy, [and] in these little islands for a black man to be independent means that he must have money or a profession".

27. "what is unique about the Caribbean is that half of it belongs to everyone else."

28. "What the stranger unacquainted with these islands must get very firmly into his head before he goes any further is that these people are not savages, they speak no other languages except English [well, not quite], they have no other religion except Christianity [again not quite], in fact, their whole outlook is that of Western civilization, modified and adapted to their particular circumstances ». Lors d'une conférence en 1969 James déclare de même qu'il n'avait pas «appris la littérature sous le manguier, ni en se baignant ou en prenant le soleil sur les rivages d'une colonie ». ("from the mango-tree, or bathing on the shore and getting the sun of the colonial countries ») Caribbean Artists Movement, Savacou, vol. 2 (Kingston, Jamaica, Caribbean Artists Movement., 1970).

29. Interview de C. L. R. James par Richard Small à Londres en Octobre 1967, reproduite dans Berman P., et al., 1981, p. 13. ( What you need in studying any historical subject is you must get some idea of the economic circumstance; you must also get some idea of the political circumstances and you must get to know the literary circumstances. Only when you know those three, you have some idea of the historical development of the period ».

30. Un soldat en Egypte écrit ainsi : « Nous ne sommes traités ni comme des chrétiens, ni comme des Britanniques, mais comme des "Nègres" antillais». («We are treated neither as Christians nor as British citizens, but as West Indian "niggers" without anybody to be interested in nor look after us ») (Rush A. S., $2011: 125$ ).

31. Ce groupe patronal fondé en 1878 existe toujours. Jusqu'à 2019 sa page de présentation réalisait la prouesse de présenter une photographie qui ne donnait à voir que des Blancs : http:// www.trinidadunionclub.com/. Consulté en septembre 2019. Le site n'est plus consultable, reste la page du réseau social : https://www.facebook.com/trinidadunionclub/

32. Ces élections étaient tout de même limitées puisque seuls sept membres sur 27 étaient désignés par le vote. Voir Høgsbjerg C., 2011 : 27. Par ailleurs James défend Cipriani face aux polémiques soulevées au sein du TWA par son refus de soutenir une loi légalisant le divorce. Ces débats conduisirent au départ de très nombreux membres du TWA, à commencer par de nombreux Trinidadiens d'origine indienne.

33. " Not the slightest trace of ill-feeling ».

34. « in these colonies today no conflict between freshly assimilated ideas of modern democracy and age-old habits based on tribal organization or a caste system ».

35. Leonard Woolf, à la tête de Hogarth Press, après avoir publié The Life, lui demande un résumé, ce sera The Case For West-India Self-Governement, un pamphlet de 32 pages qui reprend les passages de the Life en excluant tout ce qui concerne Cipriani.

36. «Cricket had plunged me into politics long before I was aware of it. When I did turn to politics, I did not have too much to learn » (Charles Forsdick and Christian Høgsbjerg, The Black Jacobins Reader, 2017, 22.).

37. «I'm afraid that American theorists will not understand this, but the clue to everything lies in his proper appreciation of the game of cricket ». 
38. "I read the History of the Russian Revolution because I was very much interested in history and the book seemed to offer some analysis of modern society. At the end of reading the book, Spring 1934, I became a Trotskyist - in my mind and later joined. It was clear in my mind that I was not going to be a Stalinist. »

39. Il ne revint à Trinidad qu'en 1958, à 57 ans, sur l'invitation du nouveau gouvernement nationaliste d'Eric Williams, le non moins fameux auteur d'Esclavage et Capitalisme, pour participer à l'inauguration du Parlement fédéral le 22 avril. Williams le convainc de rester et James dirige alors le journal du parti, The Nation. Plus tard, en 1965-1966, il est le leader d'un éphémère parti d'opposition, le Workers' and Farmers' Party. Selon Walton Look Lai, à Trinidad il est avant tout connu comme un " militant controversé des premiers temps du nationalisme » (Lai W. L. 1992 : 175).

40. «Victorian with a rebel seed».

\section{RÉSUMÉS}

Cet article propose de replacer Cyril Lionel Robert James dans le contexte caribéen, celui de sa jeunesse à Port-of-Spain. James personnifie les traversées de frontières politiques et intellectuelles, de la marge au centre et inversement, de la littérature à l'histoire et réciproquement. A partir d'un ouvrage injustement méconnu, Beyond a Boundary, un texte original à mi-chemin entre histoire du cricket et parcours intellectuel d'un jeune trinidadien, nous mettons en lumière les liens entre l'effervescence politique anticolonialiste qu'il connut à Port-of-Spain dans les années 1920, et sa formation intellectuelle. L'originalité de sa pensée apparait déjà dans ses œuvres de jeunesse : le court roman Minty Alley, écrit à la fin des années 1920, et son premier essai politique, The Life of Captain Cipriani, une biographie d'un des premiers leaders nationalistes de Trinidad, publié à Londres en 1933 par les éditions Hogarth. A partir de ces premiers écrits nous découvrons ses efforts précoces pour «Provincialiser l'Europe " pourrait-on dire, en reprenant cette expression formulée en 2000 par l'historien Dipesh Chakrabarty, et pour penser hors de l'historicisme d'une continuité historique dont le point d'aboutissement serait la modernité occidentale.

This article proposes to place Cyril Lionel Robert James in the Caribbean context, that of his youth in Port-of-Spain. James personifies the crossing of political and intellectual boundaries, from the margins to the center, from literature to history and vice versa. From an unjustly littleknown text, Beyond a Boundary, an original book halfway between the history of cricket and the intellectual journey of a young Trinidadian, we highlight the links between the anti-colonial political effervescence he experienced in Port-of-Spain in the 1920s and his intellectual training. The originality of his thought is already apparent in his early works: the short novel Minty Alley, written in the late 1920s, and his first political essay, The Life of Captain Cipriani, a biography of one of Trinidad's first nationalist leaders, published in London in 1933 by Hogarth Press. From these early writings we discover his early efforts to "Provincialize Europe", one might say, using the expression formulated in 2000 by the historian Dipesh Chakrabarty, and to think outside historicism of a historical continuity whose culmination point would be Western modernity.

Este artículo propone situar a Cyril Lionel Robert James en el contexto caribeño, el de su juventud en Puerto España. James personifica el cruce de fronteras políticas e intelectuales, de los 
márgenes al centro y viceversa, de la literatura a la historia y viceversa. De una obra injustamente incomprendida, Beyond a Boundary, texto original a medio camino entre la historia del cricket y el viaje intelectual de un joven trinitario, destacamos los vínculos entre la efervescencia política anticolonial que experimentó en Puerto España en el decenio de 1920 y su formación intelectual. La originalidad de su pensamiento se manifiesta ya en sus primeras obras: la novela corta Minty Alley, escrita a finales del decenio de 1920, y su primer ensayo político, The Life of Captain Cipriani, una biografía de uno de los primeros dirigentes nacionalistas de Trinidad, publicada en Londres en 1933 por Hogarth Editions. A partir de estos primeros escritos descubrimos sus primeros esfuerzos para "Provincializar Europa", se podría decir, utilizando la expresión formulada en 2000 por el historiador Dipesh Chakrabarty, y por pensar fuera del historicismo en una continuidad histórica cuyo punto de llegada sería la modernidad occidental.

\section{INDEX}

Mots-clés : Cyril Lionel Robert James, Atlantique noir, Post-colonialisme, Marxisme, Trinidad, Cricket

Palabras claves : Cyril Lionel Robert James, Atlántico Negro, Post-colonialismo, Marxismo, Trinidad, Cricket

Keywords : Cyril Lionel Robert James, Black Atlantic, Post-colonialism, Marxism, Trinidad, cricket

\section{AUTEUR}

\section{OLIVIER MAHEO}

Olivier Maheo, ATER en histoire (Post-doctoral assistant), Université de Poitiers, CRIHAM. Sous la direction de Mme Le Dantec- Lowry (Pr Sorbonne Nouvelle), il a soutenu sa thèse intitulée Divided we stand: Tensions et clivages au sein des mouvements de libération noire, du New Deal au Black Power, et propose une histoire des mobilisations africaines-américaines du New Deal au Black Power, au travers d'une approche politique et culturelle. Il est par ailleurs toujours rattaché à la Sorbonne Nouvelle, ED 625 MAGIIE, UA 34399, CREW. Contact olivier.maheo@univ-Poitiers.fr 